\title{
Effectiveness of Breathing Exercises on Spinal Posture, Mobility and Stabilization in Patients with Lumbar Instability
}

\author{
Jim-Yi Kang, PT, MS • Dong-Kwon Seo, PT, PhD • Ju-Chul Cho, PT, MS ${ }^{\dagger}$ Byoung-Kwon Lee, PT, PhD ${ }^{\dagger}$ \\ Department of Physical Therapy, Konyang University \\ ${ }^{1}$ Department of Rehabilitation Medicine, Wellciti Hospital
}

Received: March 1, 2018 / Revised: April 25, 2018 / Accepted: May 24, 2018

(C) 2018 J Korean Soc Phys Med

\section{| Abstract |}

PURPOSE: This study was conducted to monitor the performance of breathing exercises by patients with lumbar instability who had altered breathing patterns.

METHODS: To investigate the effects of breathing exercises on spinal posture, mobility, and stabilization in patients with lumbar instability with altered breathing patterns, 30 adult participants were enrolled on the basis of the selection criteria and randomly assigned to the breathing exercise group (BEG) or trunk stabilization exercise group (SEG). A pre-test was performed prior to the intervention exercise program. The intervention exercise program consisted of 15 sessions (three sessions per week for 5 weeks) between August and September of 2016. The post-test was performed on the 6th week of intervention.

RESULTS: Pre- and post-test comparisons of BEG and SEG revealed significant improvements in all tested items in

$\dagger$ Corresponding Author : Byoung-Kwon Lee lbk6326@konyang.ac.kr, http://orcid.org/0000-0002-1230-6088 This is an Open Access article distributed under the terms of the Creative Commons Attribution Non-Commercial License (http://creativecommons.org/licenses/by-nc/3.0) which permits unrestricted non-commercial use, distribution, and reproduction in any medium, provided the original work is properly cited. the SEG, except for spinal mobility, while significant improvements in spinal postures 1 and 2, spinal mobility, and stabilization were found in the BEG. Between-group comparisons revealed that there were no significant differences in spinal posture 1 , spinal posture 2, spinal mobility, or stabilization, whereas significant differences were found in spinal posture 2 and spinal mobility, with the BEG showing greater improvements than the SEG.

CONCLUSION: Based on the findings in the present study, it is believed that breathing exercises have important effects on spinal posture, mobility, and stabilization in patients with lumbar instability who have altered breathing patterns.

Key Words: Breathing exercise, Lumbar instability, Trunk stabilization

\section{Introduction}

Spinal segment instability refers to displacement of the mechanical axis of motion from the inside to the outside of the disc becoming more severe, thereby making it difficult for muscle activity control to maintain a stable angle or position within the support plane against displacement of the body center. Moreover, changes in the compensatory spine angle from an unbalanced muscle 
recruitment that may occur during such a time can exacerbate pain and dysfunction (Hodges and Richardson, 1999; Selles et al., 2001). Such an increase in instability causes cord level motor system imbalance while also causing pain in the lower extremities involved with activities of daily living (ADLs), such as walking and sitting (Comerford and Mottram, 2001; McConnell, 2002). Many previous studies have reported transversus abdominis dysfunction, along with a trunk muscle activation pattern that is different in patients with chronic low back pain (CLBP) or lumbar instability, which include delayed contraction of the transversus abdominis, reduced motor control ability, abnormal recruitment patterns, reduced contraction ability, and excessive activities of the rectus abdominis muscle and external oblique abdominal muscles (Ferreira et al., 2004; Urquhart et al., 2005; Hides et al., 2010).

The diaphragm cooperates with other muscles near the abdominal cavity to regulate intra-abdominal pressure (IAP). Such postural control functions of the chest cavity and abdomen through breathing by the diaphragm cooperate with other active movement systems to maintain and increase spinal and truncal mobility and stability, which have important effects on low back pain (Kalpakcioglu et al., 2009; Kolar et al., 2010; Bradley and Esformes, 2014). Moreover, cooperation between the abdominal muscles and diaphragm can reduce spinal stability and cause changes to movement patterns (Liebenson, 2004). Contraction of the transversus abdominis and pelvic floor through abdominal drawing and neutral position control by the pelvis are the most basic strategies in stabilization programs (Oliveira et al., 2017). Recently, studies have begun to investigate the improvement of cardiopulmonary function by actively using various breathing exercises based on the breathing function of the active structure of the trunk, along with changes in trunk structure and function (McConnell and Romer, 2004; Koppers et al., 2006). During maximal forced expiration, the transversus abdominis showed the highest activation changes among other abdominal muscles, while the activation change appeared greater than that of the abdominal drawing strategy (Jhu et al., 2010; Ishida et al., 2012). These effects increased the possibility of training the transversus abdominis and pelvic floor through an expiration strategy in patients with CLBP or lumbar instability.

Trunk breathing pattern changes disturb coordination of the co-contraction of active movement structures, and a single dysfunction affects other elements and subsequently causes problems in mobility and stability, including spinal posture (Wallden, 2009). The association between CLBP and instability is very high, with $23-69 \%$ of patients with CLBP having lumbar local instability (Sihvonen et al., 1997). Recently, the number of study cases investigating breathing exercises in musculoskeletal system patients has increased; however, clinical studies that apply breathing exercises in patients with lumbar instability are still lacking in Korea. Accordingly, the present study investigated application of breathing exercises for reeducation of patients with lumbar Instability who have altered breathing patterns and the effects of such exercises on the spine.

\section{Methods}

\section{Subjects}

The subjects consisted of adults residing in Gwangju, South Korea, who received explanations regarding the objectives and procedures in the study and voluntarily provided their consent to participate. 1) Adults aged 20-40 years with lumbar instability (characterized by excessive movement in specific segments during active movement and posterior-anterior spring test causing large movements and pain), 2) no history of back surgery, inflammatory spinal disorder, deformed vertebrae, fracture, or neurological radiating pain, 3) no spinal muscle strengthening and cardiopulmonary exercises for the past 1 month, 4) and no diagnosis of pathological problems 
in the respiratory system were included in this study.

\section{Process of Research}

A total of 27 adults with lumbar instability and altered breathing patterns who were selected on the basis of the selection criteria of the study were randomly assigned into an experimental group (BEG; $\mathrm{n}=13$ ) or a control group (SEG; $\mathrm{n}=14$ ). After conducting a pre-test, the intervention was applied for 5 weeks between August and September of 2016. The intervention consisted of three sessions per week for a total of 15 sessions. The post-test was performed on week 6 of the intervention. The overall exercise program for both groups consisted of $5 \mathrm{~min}$ of light treadmill walking as a warm-up exercise, followed by $30 \mathrm{~min}$ of breathing exercise for the experimental group and trunk stabilization exercise for the control group as the main exercise and then 5 min of light trunk stretching for the cool down exercise.

\section{Intervention}

A basic breathing exercise program was applied in sitting and standing postures as shown in Fig. 1 (Lehnert-Schroth, 2000; Perri, 2007). After receiving education regarding abdominal movement during inspiration and expiration, rib movement, stabilization and proper direction of the movement of the sternum, the subjects corrected problematic breathing on their own. Next, diaphragmatic breathing was facilitated by diaphragmatic compression during inspiration and relaxation during expiration. Afterwards, coordinated exercise with abdominal drawing and normal mechanism of breathing exercise were applied. The strengthening exercise method was as follows (Fig. 2). Neutral positions of the spine in the sitting and standing postures were determined, and inhalation and exhalation

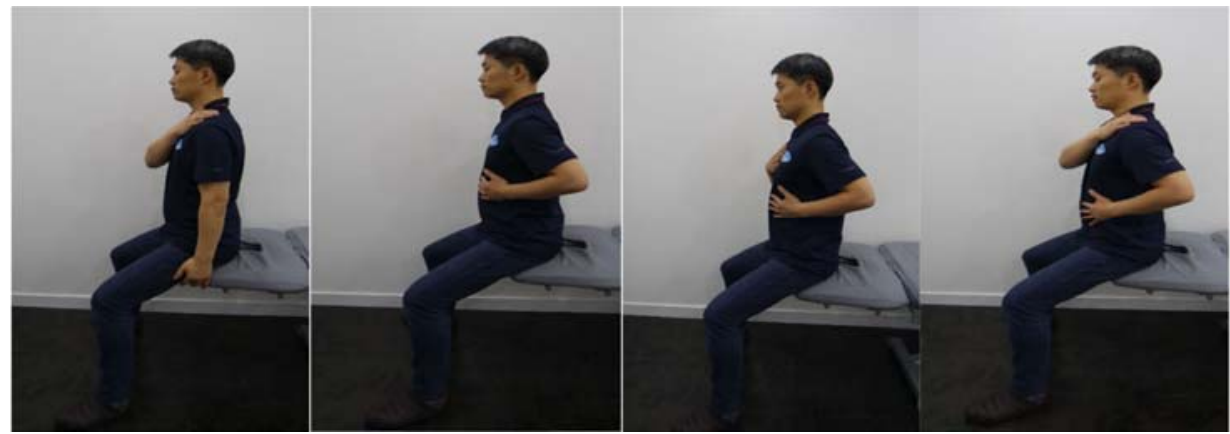

Fig. 1. Breathing correction and exercise used in this study
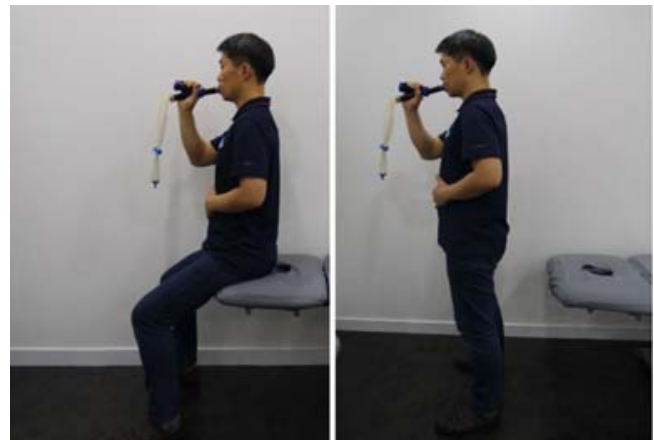

Fig. 2. Breath strengthening exercise used in this study 
were conducted with the mouthpiece of the breathing equipment in the mouth so that the exercise could strengthen the pattern of proper breathing by strengthening the actions of the respiratory muscles. If the subjects felt dizzy or fatigued during the exercise, they were allowed to rest, after which the exercise was restarted. The ventilation bag used in the exercise was adjusted in accordance with the capabilities of the respiratory muscles of each patient, while the breathing frequency was set within the range of resting respiration per minute. Prior to the experiment, the respiratory capacity for each individual was determined, and the subjects learned the exercise methods. The experimental group exercised for 30 min for each session, which was divided into three sets with each set lasting for $10 \mathrm{~min}$. A rest period of 2 min was provided between each set and the exercise was performed for 5 weeks.

The trunk stabilization exercise changed from non-resistance to external resistance (Fig. 3), the direction of resistance was changed from proximal to distal and from up and down to lateral, and the support surface was changed from non-weight bearing to weight-bearing posture. The exercise was based on the approach of relearning for reduced deep muscle control ability in patients with low back pain, and all exercises were accompanied by contraction of the pelvic floor muscles (O'Sullivan, 2000).

Basic training was performed in the dorsal recumbent, quadruped, sitting, and standing postures. The subjects

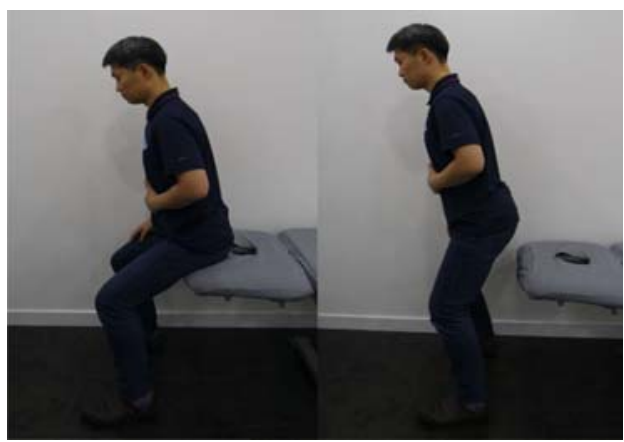

Fig. 3. Stabilization exercise used in this study trained for co-contraction of the pelvic floor and transversus abdominis, as well as determined their neutral spinal position through pelvic control without corresponding to global muscles. Contraction was maintained for 10-60 s, and the number of sets ranged between 5 and 10. Each session lasted for $30 \mathrm{~min}$, and the exercise program was applied for 2 weeks. For strengthening training, an elastic band was utilized to apply resistance in the dorsal recumbent position, while hip joint flexion and standing were applied in the sitting posture and squatting in the standing posture. The posture was maintained for 10-60 $\mathrm{s}$, while the number of sets ranged between 5 and 10. Each session lasted for $30 \mathrm{~min}$, and the exercise program was applied for 3 weeks (Ki et al., 2016).

\section{Clinical Measurement}

The SPIRO TIGER ${ }^{\circledR}$ (ldiag, Switzerland) breathing exercise equipment was used. This equipment, which allows forced breathing training, has a mouth piece and a respirator bag connected by a tube. A ventilation hole in the tube allows inflow and outflow of air to appear during breathing. The equipment is designed to allow proper outflow and inflow of $\mathrm{CO}_{2}$ and $\mathrm{O}_{2}$ to prevent dizziness from breathing (Markov et al., 2001). A Spinal Mouse (IDLAG AG, Swiss) was used for the spinal measurements. This is a device that measures spinal mobility and shape on the sagittal and frontal planes, and its reliability has been confirmed via correlation analysis based on time differences and measurers.

\section{Statistical Analysis}

Descriptive statistics were used to investigate the general characteristics of the subjects, while paired t-tests were used for the within-group comparisons of spinal posture, mobility, and stabilization between pre- and post-interventions. Independent t-tests were employed to identify differences between groups. Data analysis was conducted using the SPSS (Version 18.0; SPSS Inc., 
Table 1. General Characteristics of the Subjects

\begin{tabular}{cccc}
\hline Baseline Variable & BEG $(\mathrm{n}=13)^{*}$ & SEG $(\mathrm{n}=14)^{*}$ & $\mathrm{t}$ \\
\hline Age (years) & $26.93 \pm 6.01$ & $29.43 \pm 5.06$ & -1.19 \\
Height $(\mathrm{cm})$ & $158.50 \pm 5.78$ & $162.21 \pm 4.00$ & -1.98 \\
Weight $(\mathrm{kg})$ & $56.50 \pm 7.91$ & $61.07 \pm 7.30$ & -1.59 \\
\hline
\end{tabular}

$\mathrm{BEG}=$ breathing exercise group, $\mathrm{SEG}=$ stabilization exercise group.

*Values are expressed as the mean $\pm \mathrm{SD}$.

Chicago, IL, USA) statistical software. A $\mathrm{p}<.05$ was considered to indicate statistical significance.

\section{Result}

A total of 27 subjects who participated in the present study were randomly allocated into the BEG and SEG. In the homogeneity test, there were no significant differences between groups (Table 1). With respect to standing posture 1 , there were significant differences in both thoracic flexion angle $(p<.01)$, and lumbar extension angle $(p<.05)$ within the BEG and SEG. Comparison of the changes between groups revealed no significant differences in the thoracic or lumbar areas. With respect to standing posture 2 (with external load), there were significant differences in the thoracic flexion angle decrease (extension) and lumbar extension angle decrease (flexion) $(p<.01)$ within the BEG and SEG. Additionally, comparison of the changes between groups revealed significant differences in the lumbar area, but not in the thoracic area $(p<.01)$. With respect to spinal mobility, there were significant differences in the thoracic flexion mobility increase and lumbar flexion mobility decrease within the BEG $(p<.01)$. Moreover, the thoracic and lumbar flexion mobility increased and decreased within the SEG, respectively; however, the differences were not significant. Comparison of the changes between groups revealed significant differences in the thoracic flexion mobility increase $(p<.01)$; however, the lumbar flexion mobility decrease was not significant. With respect to spinal stabilization, there were significant differences in the compensatory sagittal plane angle decrease for thoracic flexion and lumbar extension $(p<.01)$ within the BEG and SEG. Comparison of the changes between groups revealed no significant differences in the thoracic or lumbar areas (Table 2).

\section{Discussion}

The present study applied breathing exercises to patients with lumbar instability who had altered breathing patterns and measured their spinal posture, segment mobility, and spinal stabilization ability with loads. The study also aimed to verify the importance of breathing exercises for patients with lumbar instability and CLBP by comparing the results to those of trunk stabilization exercises, which have been confirmed to be effective for treating spine curvature changes, as well as inducing spinal mobility and stabilization in patients with CLBP.

An operating force line in forward and downward directions in the lumbo-pelvic area from pelvic high, forward, and upward directions in the thoraco-abdominal area and upward direction of the head helps create a balanced spine posture (Chaitow, 2002; Otman et al., 2005). In general, the operating force line in the extension direction acts on the thoracic area, and forced breathing exercises with controlled respiratory rate and speed create extension of the ribs and facet joints in a coordinated manner (Neumann, 2013), which further induces changes in the trunk muscles. Therefore, the results observed in the th 
Table 2. Comparison of the outcome measures within and between groups

\begin{tabular}{|c|c|c|c|c|c|}
\hline & Group/Outcome & Baseline $^{\dagger}$ & 5-week ${ }^{\dagger}$ & $t$ & $t$ \\
\hline \multirow{12}{*}{ Thoracic } & Spinal Posture 1 & & & & \multirow{4}{*}{-1.37} \\
\hline & BEG & $40.77 \pm 3.72$ & $36.54 \pm 3.86$ & $9.59 * *$ & \\
\hline & SEG & $43.00 \pm 6.05$ & $39.07 \pm 5.49$ & $4.68 * *$ & \\
\hline & Spinal Posture 2 & & & & \\
\hline & BEG & $43.46 \pm 4.29$ & $39.38 \pm 4.68$ & $11.13^{* *}$ & \multirow{2}{*}{1.52} \\
\hline & SEG & $41.14 \pm 5.78$ & $35.93 \pm 6.82$ & $7.84 * *$ & \\
\hline & Spinal Mobility & & & & \multirow{3}{*}{$5.44 * *$} \\
\hline & BEG & $9.23 \pm 2.48$ & $15.62 \pm 3.52$ & $-9.08 * *$ & \\
\hline & SEG & $13.00 \pm 2.28$ & $14.07 \pm 3.17$ & -1.88 & \\
\hline & \multicolumn{4}{|l|}{ Stabilization } & \\
\hline & BEG & $4.23 \pm 2.00$ & $1.38 \pm .96$ & $4.85^{* *}$ & \multirow{2}{*}{-1.62} \\
\hline & SEG & $4.00 \pm 1.92$ & $2.07 \pm 1.20$ & $4.40 * *$ & \\
\hline \multirow{12}{*}{ Lumbar } & Spinal Posture 1 & & & & \multirow{4}{*}{-.61} \\
\hline & BEG & $-29.62 \pm 4.29$ & $-25.54 \pm 4.57$ & $-11.13^{* *}$ & \\
\hline & SEG & $-27.07 \pm 7.45$ & $-24.00 \pm 7.87$ & $-2.24 *$ & \\
\hline & Spinal Posture 2 & & & & \\
\hline & BEG & $-32.00 \pm 3.80$ & $-27.54 \pm 3.77$ & $-14.27 * *$ & \multirow{2}{*}{$-3.05 * *$} \\
\hline & SEG & $-27.21 \pm 5.25$ & $-22.79 \pm 4.26$ & $-6.11 * *$ & \\
\hline & Spinal Mobility & & & & \multirow{3}{*}{-1.85} \\
\hline & BEG & $51.00 \pm 9.78$ & $45.08 \pm 9.71$ & $5.32 * *$ & \\
\hline & SEG & $51.50 \pm 7.52$ & $49.29 \pm 5.60$ & 1.57 & \\
\hline & \multicolumn{4}{|l|}{ Stabilization } & \\
\hline & BEG & $-2.85 \pm 1.51$ & $.08 \pm 1.80$ & $-6.56^{* *}$ & \multirow{2}{*}{1.30} \\
\hline & SEG & $-3.07 \pm 2.33$ & $-.86 \pm 1.91$ & $-3.91 * *$ & \\
\hline
\end{tabular}

${ }^{\dagger}$ Values are expressed as the mean $\pm \mathrm{SD}$.*and** indicate significant differences at $p<.05$ and $p<.01$, respectively. Spinal Posture 1=standing position; Spinal Posture 2=standing position with an external load, Spinal Mobility=trunk flexion, Stabilization=standing position with an external load, BEG=breathing exercise group, $\mathrm{SEG}=$ stabilization exercise group.

oracolumbar area for spinal postures 1 and 2 in the present study indicate that pelvic control education and correction of breathing mechanism, together with coordination and strengthening of the diaphragm and abdominal muscles, had a favorable effect on balancing the trunk posture. In particular, the between-group comparison for spinal posture 2 showed significant differences in the lumbar area. It is believed that this was the result of correction of the breathing pattern as a result of the costo-diaphragmatic breathing mechanism helping to improve mobility in the sternum and ribs, which facilitated thoracic extension so that the extension motion in the lumbar area could be reduced through relative compensation. Moreover, it also increased the IAP from diaphragmatic depression and stimulated various abdominal muscles, which supported the lumbar area to reduce compensatory extension motions according to the load being applied. Since it has been reported that breath holding and trunk muscle activation 
pattern changes become more severe in patients with CLBP when an external load is applied (Perri, 2007), it was important to train the subjects to not hold their breath through breathing correction and control training when an external load was being applied. In the present study, there were significant differences in spinal mobility within the BEG, with an increased thoracic flexion mobility and decreased lumbar flexion mobility. There was also increased thoracic flexion mobility and decreased lumbar flexion mobility within the SEG; however, these differences were not statistically significant. An increased thoracic mobility through breathing exercises reported in a previous study supported the findings of the present study (Ekstrum et al., 2009). It is believed that maintaining proper muscle length and elasticity from adequate use of the respiratory muscles through recovery of proper breathing mechanisms induced thoracic relaxation, which ultimately increased the thoracic motion. Furthermore, chest relaxation and increased mobility decreased the lumbar motion during flexion. In the present study, the spinal stabilization test revealed significant decreases in the compensatory sagittal angles of thoracic flexion and lumbar extension within both groups. Comparison of the amount of changes between groups revealed no significant differences in the thoracic and lumbar angles. The present study attempted to identify changes in the trunk muscle activation patterns indirectly in patients with lumbar instability by measuring the amount of compensated angles created by the thoracic and lumbar areas. Moreover, breathing exercises were used to induce decreases in the compensated angle change to determine improvements in the stabilization ability of the spine. A significant decrease in the compensated angle was found after the breathing exercises, similar to the significant effect on compensated angles observed after trunk stabilization exercises. Perri (2007) stated that abnormal breathing patterns must be corrected to achieve postural stability and spinal stabilization. Accordingly, it is believed that correction of breathing patterns and strengthening exercises improved spinal stabilization while an external load was being applied.

The most important limitation of this study is the small sample size. In addition, it is not clear how long the training effect lasted as no follow-up was performed. Consequently, it is difficult to generalize the results of this study to all lumbar instability patients. Therefore, future studies should be conducted to overcome these limitations. We suggest that breathing exercises be considered an important intervention in clinical practice when lumbar instability treatments are planned.

\section{Conclusion}

Based on the findings of the present study, it is believed that breathing exercises have an important effect on the spinal posture, mobility, and stabilization of patients with lumbar instability who have altered breathing patterns. Considering the effects of breathing on physical and mental health, continued clinical studies of breathing in patients with various chronic musculoskeletal diseases are warranted.

\section{References}

Bradley H, Esformes J. Breathing pattern disorders and functional movement. Int J Sports Phys Ther. 2014; 9(1):28-39.

Chaitow L, Bradley D, Gilbert C. Multidisciplinary approaches to breathing pattern disorders. New York. Churchill Livingstone. 2002.

Comerford MJ, Mottram SL. Movement and stability dysfunction--contemporary developments. Man Ther. 2001;6(1):15-26.

Ekstrum JA, Black LL, Paschal KA. Effects of a thoracic mobility and respiratory exercise program on pulmonary function and functional capacity in older adults. Physical \& Occupational Therapy in Geriatrics. 
2009;27(4):310-27.

Ferreira PH, Ferreira ML, Hodges PW. Changes in recruitment of the abdominal muscles in people with low back pain: ultrasound measurement of muscle activity. Spine (Phila Pa 1976). 2004;29(22):2560-6.

Hides JA, Boughen CL, Stanton WR, et al. A magnetic resonance imaging investigation of the transversus abdominis muscle during drawing-in of the abdominal wall in elite Australian Football League players with and without low back pain. J Orthop Sports Phys Ther. 2010;40(1):4-10.

Hodges PW, Richardson CA. Transversus abdominis and the superficial abdominal muscles are controlled independently in a postural task. Neurosci Lett. 1999;265(2):91-4.

Ishida H, Hirose R, Watanabe S. Comparison of changes in the contraction of the lateral abdominal muscles between the abdominal drawing-in maneuver and breathe held at the maximum expiratory level. Man Ther. 2012;17(5):427-31.

Jhu JL, Chai HM, Jan MH, et al. Reliability and relationship between 2 measurements of transversus abdominis dimension taken during an abdominal drawing-in maneuver using a novel approach of ultrasound imaging. J Orthop Sports Phys Ther. 2010;40(12): 826-32.

Kalpakcioglu B, Altinbilek T, Senel K. Determination of spondylolisthesis in low back pain by clinical evaluation. J Back Musculoskelet Rehabil. 2009; 22(1):27-32.

Ki C, Heo M, Kim HY, et al. The effects of forced breathing exercise on the lumbar stabilization in chronic low back pain patients. J Phys Ther Sci. 2016;28(12): 3380-3.

Kolar P, Sulc J, Kyncl M, et al. Stabilizing function of the diaphragm: dynamic MRI and synchronized spirometric assessment. J Appl Physiol (1985). 2010;109(4): 1064-71.
Koppers RJ, Vos PJ, Boot CR, et al. Exercise performance improves in patients with COPD due to respiratory muscle endurance training. Chest. 2006;129(4): 886-92.

Lehnert-Schroth C. Three-dimensional treatment for scoliosis: a physiotherapeutic method for deformities of the spine. Martindale Pr. 2000.

Liebenson C. Spinal stabilization-an update. Part 1biomechanics. Journal of bodywork and movement therapies. 2004;8(2):80-4.

Markov G, Spengler CM, Knopfli-Lenzin C, et al. Respiratory muscle training increases cycling endurance without affecting cardiovascular responses to exercise. Eur J Appl Physiol. 2001;85(3-4):233-9.

McConnell AK, Romer LM. Respiratory muscle training in healthy humans: resolving the controversy. Int J Sports Med. 2004;25(4):284-93.

McConnell J. Recalcitrant chronic low back and leg pain--a new theory and different approach to management. Man Ther. 2002;7(4):183-92.

Neumann DA. Kinesiology of the Musculoskeletal SystemE-Book: Foundations for Rehabilitation. Elsevier Health Sciences. 2013.

Oliveira CB, Negrao Filho RF, Franco MR, et al. Psychometric Properties of the Deep Muscle Contraction Scale for Assessment of the Drawing-in Maneuver in Patients With Chronic Nonspecific Low Back Pain. J Orthop Sports Phys Ther. 2017;47(6):432-41.

O'Sullivan PB. Lumbar segmental 'instability': clinical presentation and specific stabilizing exercise management. Man Ther. 2000;5(1):2-12.

Otman S, Kose N, Yakut Y. The efficacy of Schroth s 3-dimensional exercise therapy in the treatment of adolescent idiopathic scoliosis in Turkey. Saudi Med J. 2005;26(9):1429-35.

Perri M. Rehabilitation of breathing pattern disorders. Rehabilitation of the Spine: a Practitioners Manual. USA. Lippincott Williams \& Wilkins. 2007. 
Selles RW, Wagenaar RC, Smit TH, et al. Disorders in trunk rotation during walking in patients with low back pain: a dynamical systems approach. Clin Biomech (Bristol, Avon). 2001;16(3):175-81.

Sihvonen T, Lindgren KA, Airaksinen O, et al. Movement disturbances of the lumbar spine and abnormal back muscle electromyographic findings in recurrent low back pain. Spine (Phila Pa 1976). 1997;22(3):289-95.

Urquhart DM, Barker PJ, Hodges PW, et al. Regional morphology of the transversus abdominis and obliquus internus and externus abdominis muscles. Clin Biomech (Bristol, Avon). 2005;20(3):233-41.

Wallden M. The neutral spine principle. J Bodyw Mov Ther. 2009;13(4):350-61. 